\title{
Targeted deletion of matrix metalloproteinase-9 attenuates left ventricular enlargement and collagen accumulation after experimental myocardial infarction
}

\author{
Anique Ducharme, ${ }^{1}$ Stefan Frantz, ${ }^{1}$ Masanori Aikawa, ${ }^{1}$ Elena Rabkin, ${ }^{2}$ \\ Merry Lindsey, ${ }^{1}$ Luis E. Rohde, ${ }^{1}$ Frederick J. Schoen, ${ }^{2}$ Ralph A. Kelly, ${ }^{1}$ Zena Werb, ${ }^{3}$ \\ Peter Libby, ${ }^{1}$ and Richard T. Lee ${ }^{1}$ \\ ${ }^{1}$ Cardiovascular Division, Department of Medicine, and \\ ${ }^{2}$ Department of Pathology, Brigham and Women's Hospital, Harvard Medical School, Boston, Massachusetts, USA \\ ${ }^{3}$ Department of Anatomy, University of California, San Francisco, California, USA
}

Address correspondence to: Richard T. Lee, Cardiovascular Division, Brigham and Women's Hospital, 75 Francis Street, Boston, Massachusetts 02115, USA. Phone: (617) 732-7146; Fax: (617) 264-5139; E-mail: rlee@rics.bwh.harvard.edu.

Received for publication October 25, 1999, and accepted in revised form May 17, 2000.

\begin{abstract}
Matrix metalloproteinase-9 (MMP-9) is prominently overexpressed after myocardial infarction (MI). We tested the hypothesis that mice with targeted deletion of MMP9 have less left ventricular (LV) dilation after experimental MI than do sibling wild-type (WT) mice. Animals that survived ligation of the left coronary artery underwent echocardiographic studies after MI; all analyses were performed without knowledge of mouse genotype. By day 8, MMP9 knockout (KO) mice had significantly smaller increases in end-diastolic and end-systolic ventricular dimensions at both midpapillary and apical levels, compared with infarcted WT mice; these differences persisted at 15 days after MI. MMP-9 KO mice had less collagen accumulation in the infarcted area than did WT mice, and they showed enhanced expression of MMP-2, MMP-13, and TIMP-1 and a reduced number of macrophages. We conclude that targeted deletion of the $M M P 9$ gene attenuates LV dilation after experimental MI in mice. The decrease in collagen accumulation and the enhanced expression of other MMPs suggest that MMP-9 plays a prominent role in extracellular matrix remodeling after MI.
\end{abstract}

J. Clin. Invest. 106:55-62 (2000).

\section{Introduction}

The extent of ventricular dilation after a myocardial infarction (MI) depends on the magnitude of the initial ischemic damage as well as the tissue healing process $(1,2)$. This dynamic pathological process includes the expression and activation of the matrix metalloproteinases (MMPs), which may mediate many of the morphological changes that occur after MI in both infarcted and noninfarcted regions (3-5). Members of the MMP family of enzymes degrade specific extracellular matrix components; clinical and experimental studies have shown that MMP expression and activity increase in both MI (5) and dilated cardiomyopathy $(6,7)$.

Because extracellular matrix deposition and organization play a major role in left ventricular (LV) remodeling, MMP inhibition has emerged as a potential therapeutic strategy for patients at risk for the development of congestive heart failure. Administration of a broad-spectrum MMP inhibitor attenuates $\mathrm{LV}$ enlargement in pacing-induced congestive heart failure (8) and in the early period after MI occurs (9). Whether this effect depends on the inhibition of many MMPs, or selective inhibition of MMPs can prevent ventricular dilation remains unexplored. Recently, Heymans et al. reported a decreased incidence of rupture at 4 days after MI in MMP-9-deficient animals, suggesting that MMP-9 may have a specific role in early myocardial healing (10). In this study we evaluated the influence of targeted deletion of the MMP-9 gene on $\mathrm{LV}$ remodeling after experimental $\mathrm{MI}$ in mice. We performed this study with sibling wild-type (WT) controls after at least six backcrosses to minimize genetic variability. All analyses were blinded to genotype; the animals were studied for 15 days.

\section{Methods}

Animals and surgery. We used the progeny of heterozygous breeding pairs of mice with targeted disruption of MMP-9, as described by Vu et al. (11). MMP-9-deficient mice have delayed long-bone growth and development due to delayed angiogenesis and ossification; however, by adulthood, these changes result in only a $10 \%$ shortening in the long bones. Animals with an FVB background were backcrossed; our studies used the homozygous MMP-9-deficient and sibling WT offspring of generation six or higher. Offspring were eartagged and coded, with tail DNA samples harvested for genotyping using PCR. For MMP-9, we used a sense oligonucleotide primer (5'-GCA TAC TTG TAC CGC TAT GG - $3^{\prime}$ ) and an antisense primer (5'-TAA CCG GAG GTC CAA ACT GG-3'). For the neomycin cassette, we also used a sense oligonucleotide primer (5'-GAA 
GGG ACT GGC TGC TAT TG-3') and an antisense primer (5'-AAT ATC ACG GGT AGC CAA CG-3'). Again, all procedures were performed without knowledge of genotype. Males with deletion of MMP-9 and WT males, ranging in age from 8 weeks to 10 weeks, and in weight from 25 grams to 30 grams, underwent coronary artery ligation for the production of MI. Surgical procedures have been described in detail elsewhere (9, 12). Briefly, after anesthesia with pentobarbital $(25-30 \mu \mathrm{g} / \mathrm{g}$ intraperitoneally) and intubation with a polyethylene tube (size 60), animals were ventilated with a volume-cycled rodent respirator (Harvard Apparatus Co., South Natick, Massachusetts, USA) with a $2-3 \mathrm{~mL} / \mathrm{cycle}$ at a respiratory rate of 115 cycles/min. After thoracotomy, ligation of the left coronary artery was performed with a 7-0 silk suture, 3-4 $\mathrm{mm}$ from the tip of the left auricle. Pallor, regional hypokinesia, and enlargement of the left ventricle confirmed the presence of an infarction. The chest wall was closed with a continuous $6-0$ prolene suture, and the skin was closed with 4-0 polyester sutures. The animals were then extubated and allowed to recover from surgery under a heating lamp for 1 hour. Antibiotic prophylaxis was not given, but no apparent infection developed in any animal during the course of the study or at the time of autopsy. All mice were housed under identical conditions, and were given food and water ad libitum. The Harvard Medical School Standing Committee on Animal Research approved the study protocol.

Echocardiographic imaging. Echocardiographic studies were performed under light anesthesia with spontaneous respiration using intraperitoneal 2,2,2-tribromoethanol (Avertin; Aldrich, Milwaukee, Wisconsin, USA) in a $2.5 \% \mathrm{wt} / \mathrm{vol}$ solution $(8 \mu \mathrm{L} / \mathrm{g}$ of mouse $)$ as previously described (9). An ultrasonographer experienced in rodent imaging performed the echocardiographic studies, using commercially available equipment (Sonos 5500; Hewlett Packard Medical Products, Andover, Massachusetts, USA) and an 8- to $12-\mathrm{MHz}$ transducer. Dynamically focused annular array and fusion frequency technologies were used, allowing ultrasound frequencies of up to $18 \mathrm{MHz}$. A standoff was used, depth was set at $4 \mathrm{~cm}$, and the zoom mode was used to optimize resolution and penetration. Frame acquisition rates using the loop mode reached up to $120 \mathrm{MHz}$, allowing excellent temporal resolution. Identical zoom size and depth settings were used between examinations to facilitate calibration for offline analysis.

M-mode images were obtained at a sweep speed of $100 \mathrm{~mm} / \mathrm{s}$. Two-dimensional image-guided M-mode recordings were made at the midpapillary level. Apical image-guided M-mode recordings were obtained at the greatest area/diameter in the lower third of the left ventricle. The images were recorded on S-VHS tapes for offline analysis. Echocardiographic studies were performed at baseline (18-24 hours after the surgical procedure), at 4 days, at 8 days, and at 15 days after the surgery, immediately before the sacrifice of the animals. We allowed an 18- to 24-hour recovery period to minimize the residual negative inotropic and chronotropic effects that may be encountered after general anesthesia (furthermore, quality of echocardiographic imaging is not optimal immediately after a thoracotomy). Echocardiographic acquisition and analysis was performed by a single echocardiographer blinded to mouse genotype. Enddiastolic and end-systolic diameters were measured, and fractional shortening was calculated. For each measurement, three consecutive cardiac cycles were measured and averaged.

Tissue collection. Mice were sacrificed after the last echocardiographic study, on day 15 . Hearts were excised, and the right and left ventricles were separated. A transverse section $(5-7 \mathrm{~mm})$ was obtained at the midventricular level. Tissue sections were embedded in OCT compound (Sakura, Torrance, California, USA) and frozen in 2-methylbutane prechilled with liquid nitrogen. Tissue blocks were stored at $-80^{\circ} \mathrm{C}$ until sectioning. In a subset of eight animals (4 MMP-9 KO and 4 WT), the left ventricle was divided between the apical and papillary regions and frozen in liquid nitrogen for immunoblot analysis.

Northern and Western blots. Total cellular RNA was isolated by a modification of the acid guanidinium thiocyanate and phenol/chloroform extraction method, using TRIzol LS Reagent (Life Technologies Inc., Gaithersburg, Maryland, USA) according to the manufacturer's protocol. RNA was size fractionated on 1.0\% agarose, transferred to a nylon membrane, and crosslinked with ultraviolet radiation. A murine collagen type I $\alpha$ cDNA was radiolabeled using a random primer labeling kit (Life Technologies Inc.). Normalization of RNA for equal loading was carried out by rehybridizing with a murine GAPDH cDNA probe. Autoradiographs were scanned using version 1.62 of the Image program from the National Institutes of Health.

For Western analysis, frozen tissue was homogenized in buffer containing 1\% nonylphenol ethoxylate, $0.5 \%$ sodium deoxycholate, $0.1 \%$ SDS, and protease inhibitors in PBS. Equal amounts of the denatured protein from the supernatant were loaded per lane for SDS-PAGE. After running the gel, proteins were transferred to a PVDF membrane. After blocking with 5\% nonfat dry milk in Tris-buffered saline with $0.1 \%$ Tween 20 , membranes were incubated with antibody against MMP-2, MMP-3, TIMP-1 (Oncogene; Cambridge, Massachusetts, USA), or MMP-13 (Chemicon; Temecula, California, USA) for 60 minutes, followed by washing and incubation with a matching secondary antibody (Bio-Rad Laboratories Inc., Hercules, California, USA). Membranes were then incubated with a chemiluminescent agent (Renaissance; DuPont NEN, Boston, Massachusetts, USA) and autoradiographed.

Immunocytochemistry. Fresh-frozen sections ( $6 \mu \mathrm{m})$ were fixed with fresh $4 \%$ paraformaldehyde for 10 minutes and rinsed in PBS. Tissue sections were 
preincubated with $0.3 \%$ hydrogen peroxide in PBS to inhibit endogenous peroxidase activity, and then incubated with primary antibodies diluted in PBS, supplemented with $4 \%$ of the species-respective normal serum for 30 minutes at room temperature. After washing with PBS, species-appropriate biotinylated secondary antibodies were applied, followed by avidin-peroxidase complexes (Vector Laboratories, Burlingame, California, USA). The reaction was visualized with 3-amino-9-ethyl carbazole as substrate (AEC; Sigma Chemical Co., St. Louis, Missouri, USA), and counterstained with Gill's hematoxylin solution. The following primary antibodies were used: antimouse neutrophils (Cedarlane Laboratories, Hornby, Ontario, Canada) and antibodies against human $\alpha$ smooth muscle actin (DAKO Corp., Carpinteria, California, USA), mouse macrophage Mac-3, and human endothelial cell CD31, CD4, and CD8 (PharMingen, San Diego, California, USA). For each section, cells positive for $\mathrm{CD} 4, \mathrm{CD} 8$, and $\mathrm{CD} 31$ were counted in the infarcted area of mouse heart in at least 7-10 random high-power fields. To analyze cells that stained positive for Mac3 and $\alpha$-smooth muscle actin, a computer-based image analysis was used (see below). Cell counting and quantitative morphometry were done by two observers who were unaware of genotype.

Collagen content. Picrosirius red polarization microscopy was performed for detection of interstitial collagen according to Junqueira's method with our modifications $(13,14)$. Birefringence under illumination with polarized light identifies collagen, including types I and III (13). Sections from a subset of 15 mice (7 MMP$9 \mathrm{KO}$ and $8 \mathrm{WT}$ ) that survived the 15-day protocol were chosen; MI sizes were qualitatively similar by Masson's trichrome and picrosirius red staining. Fresh-frozen sections $(6 \mu \mathrm{m})$ were fixed in $10 \%$ neutral buffered formalin, rinsed with distilled water, and incubated with $0.1 \%$ picrosirius red F3BA (Polysciences Inc., Warren, Pennsylvania, USA) in saturated picric acid for $90 \mathrm{~min}-$ utes. Sections were rinsed twice with $0.01 \mathrm{~N} \mathrm{HCl}$ for 1 minute, and then immersed in distilled water. After dehydration with $70 \%$ ethanol for 30 seconds, sections were visualized under polarized light and photographed with the same exposure time for each section as previously described (14). Two predefined regions (infarcted and noninfarcted) were chosen and photographed at low power $(\times 40)$. A microplate reader-based quantitation of collagens was performed as described by others $(15,16)$. Briefly, collagen standards and lysates were plated on microtiter wells in triplicate, and dried onto the plates. The wells were stained with $0.1 \%$ Sirius red F3BA in saturated picric acid for 1 hour, and washed with $10 \mathrm{mM} \mathrm{HCl}$ and $0.1 \mathrm{mM} \mathrm{NaOH}$. Absorbance was read at $540 \mathrm{~nm}$. Previously, this test has been shown to be comparable to the colorimetric hydroxyproline assay (16).

Quantitative analysis for bistology. Analysis of immunohistochemistry for macrophages, neutrophils, $\alpha$-actin, and picrosirius red staining was performed with a computer-based quantitative 24-bit (16.2 million unique combinations) color image analysis system (Optimas 5.2; Optimas Corp., Bothell, Washington, USA) (17). Micrographs were scanned into a $1,000 \times$ 1,000 image buffer. A color threshold mask for immunostaining was defined to detect the red color by sampling, and the same threshold was applied to all specimens. The percentage of the total area with positive color for each section was recorded. For picrosirius red staining, a negative background (black) was chosen for thresholding, and the positive area was calculated by subtraction. Collagen volume fraction was calculated as the sum of stained tissue divided by the sum of muscle area and connective tissue in the visual field of the section. This approach predicts the proportion of myocardium occupied by fibrillar collagen, and correlates closely with the hydroxyproline concentration of the tissue (18).

Statistical analysis. In a previous study, we found that some mice that underwent surgical ligation of the left coronary artery, despite paleness and bulging of the left ventricle at the time of surgery, did not develop either wall-motion abnormalities that were visible by echocardiography or histologic evidence of infarction. Not surprisingly, these animals do not demonstrate a progressive increase in LV dimensions (9). Based on these observations, our study was designed to include in the statistical analysis only the animals that revealed a wallmotion abnormality during the baseline study, within 24 hours of the surgical procedure. This determination was made at the time of the initial echocardiogram without knowledge of mouse genotype.

The mortality data (deaths occurring soon after surgery and during the 15-day protocol, including causes of death) were analyzed with the Pearson $\chi^{2}$ test. Twoway repeated-measures mixed-model ANOVA was used to test differences between groups regarding the evolution across time of ventricular dilation. When a possible interaction was found $(P<0.25)$, slice effects (also known as simple effects) were analyzed, i.e., time effect was analyzed for each genotype group, and differences between the groups were analyzed for each

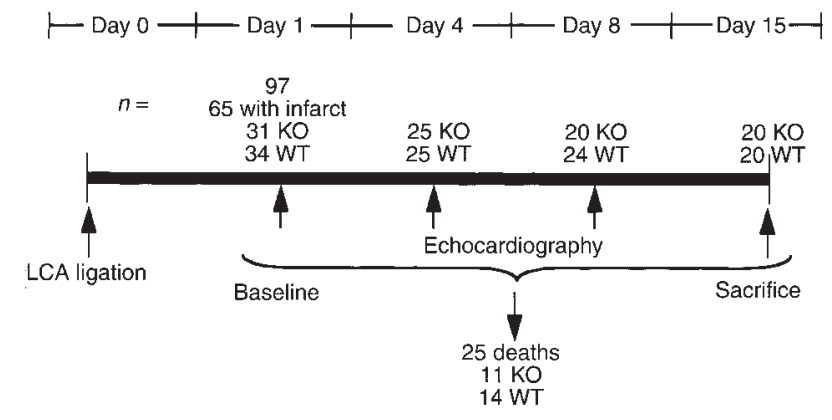

Figure 1

Design of the protocol, with timing of experimental MI, echocardiographic studies, and sacrifice. LCA, left coronary artery. KO, MMP$9 \mathrm{KO}$ animals; WT, congenic MMP-9 WT animals. 
a
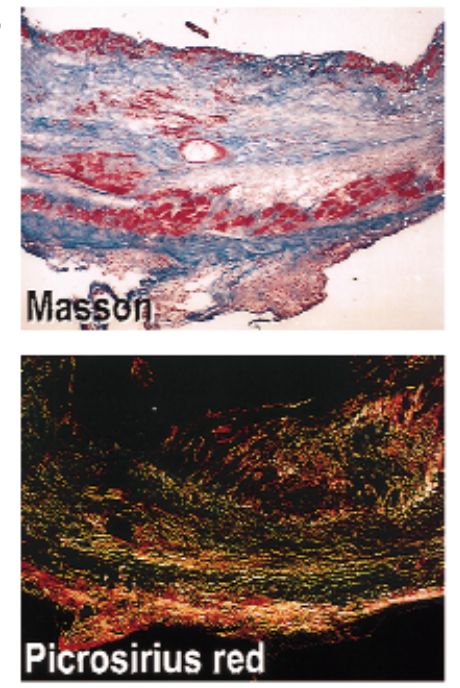
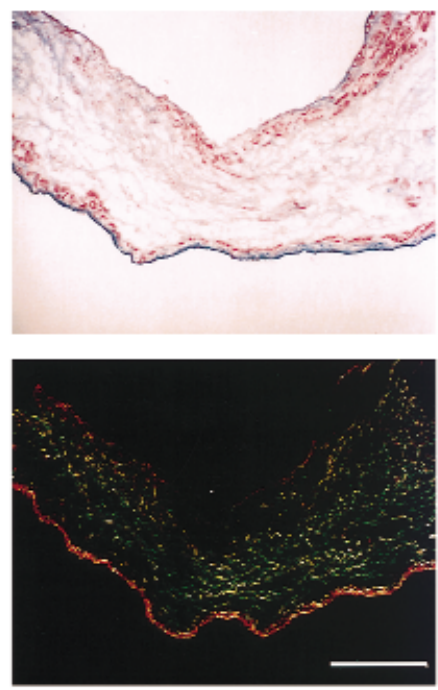

b

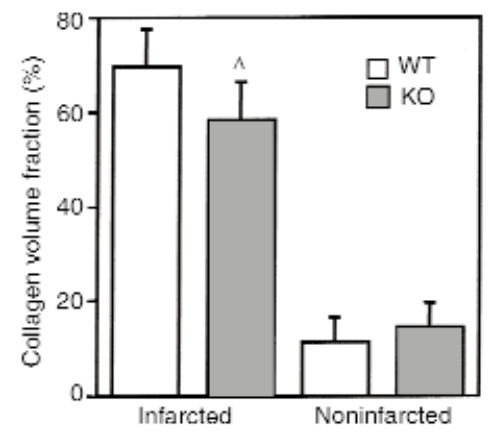

c

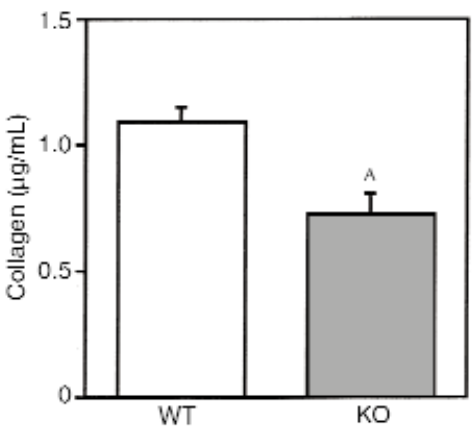

Figure 2

(a) Representative examples of staining with Masson's trichrome and picrosirius red under polarized light of the infarcted region, 15 days after experimental MI (WT is represented by the left panels, MMP-9 KO by the right panels). Picrosirius red staining demonstrated the characteristic bright yellow of collagen fibrils under polarized light, with less deposition of picrosirius red-stained collagen in MMP-9 KO samples than in WT samples (scale bar: $200 \mu \mathrm{m}$ ). (b) Collagen volume fraction from picrosirius red-stained myocardium as percentage of stained tissue in muscle areas and connective tissue in the visual field of the section. (c) Collagen content of apical myocardium after MI in infarct size matching WT and $\mathrm{KO}$ animals. ${ }^{\mathrm{A} P}<0.05$.

level of time. To keep the overall statistical significance at $5 \%$, the levels of significance for testing simple time effects were adjusted according to the Bonferroni rule. For comparisons over time after MI, repeated measures ANOVA was used. To account for the three within-group comparisons, $P<0.0167$ was considered significant. For between-group comparisons at each timepoint, a less conservative level was used; $P<0.05$ was considered significant. All the analyses were performed with the MIXED procedure of SAS 6.12 (SAS Institute Inc., Cary, North Carolina, USA) to handle missing data. All values are expressed as mean \pm SD.

\section{Results}

Operative mortality and baseline measurements. Ninetyseven mice survived the surgical procedure (56 WT and $41 \mathrm{KO}$ ); early operative mortality (within 24 hours) was $11 \%$ ( 7 WT and 4 MMP-9 KO; $P=0.67$ ). The 86 animals that survived 24 hours after surgery underwent transthoracic echocardiography for baseline imaging (Figure 1, Table 1). Infarction, as defined by wall-motion abnormality, was achieved in $78 \%$ of the procedures $(n=65,34 \mathrm{WT}$ and $31 \mathrm{MMP}$ $9 \mathrm{KO})$. Among the infarcted animals, 25 deaths (38.5\%) occurred during the 15-day protocol (14 WT and 11 MMP-9 KO; $P=0.91$ ). All mice that died and were included in the MI group were confirmed to have MI by postmortem examination. Death was attributed to congestive heart failure or arrhythmia (or both) in all cases, except for five animals that died from LV rupture, as defined by extensive blood surrounding the heart or filling the chest cavity (4 WT and $1 \mathrm{KO} ; P=0.28$ ). Baseline echocardiographic measurements of infarcted mice were similar in both groups. There was a small but statistically significant increase in LV end-diastolic dimension at baseline between the MMP-9 KO and WT groups. Based on previous data suggesting that larger ventricles at baseline are more likely to dilate with time (9), this represented a modest bias toward the null hypothesis; that is, the MMP-9 KO group would be more likely to have progressive enlargement.

Followup echocardiographic measurements (Table 2). Four days after MI, the two groups exhibited similar increases in end-systolic and end-diastolic diameters compared with baseline measurements. In contrast, 8 days after MI, both midpapillary and apical end-diastolic and end-systolic diameters increased in the WT group, with negligible changes in the MMP-9 KO mice. This attenuation of enlargement in MMP-9 KO mice continued at 15 days. During the 15-day protocol, resting heart rate increased from baseline over time in both groups, but there were no significant differences between the two groups.

Animals with no obvious cardiac enlargement or wall-motion abnormalities during the baseline echocardiogram were not included in the statistical 
Table 1

Baseline echocardiographic measurements of infarcted mice

\begin{tabular}{lcc}
\hline & MMP-9 KO & WT \\
& $n=31$ & $n=34$ \\
Midpapillary measurements & & \\
ED diameter (mm) & $2.8 \pm 0.3$ & $2.6 \pm 0.3^{\mathrm{A}}$ \\
ES diameter (mm) & $1.8 \pm 0.4$ & $1.6 \pm 0.4$ \\
M-mode FS (\%) & $37.3 \pm 9.2$ & $38.3 \pm 12.5$ \\
Apical measurements & & \\
ED diameter (mm) & $2.8 \pm 0.3$ & $2.7 \pm 0.4$ \\
ES diameter (mm) & $2.1 \pm 0.5$ & $1.9 \pm 0.6$ \\
M-mode FS (\%) & $24.5 \pm 11$ & $29.5 \pm 16$ \\
\hline
\end{tabular}

ED, end-diastolic; ES, end-systolic; FS, fractional shortening. Data are expressed as mean $\pm S D$. ${ }^{A} P<0.01$; otherwise, there were no significant differences between MMP-9 KO mice and WT mice at baseline.

analysis, but were followed for the 15-day protocol and analyzed separately $(n=21)$. There were 16 "unintended shams" in the WT group, and 5 in the MMP-9 KO group $(P<0.001)$. These mice showed no ventricular dilation during the 15-day protocol (data not shown).

Collagen analysis. Masson's trichrome staining and picrosirius red polarization for collagen was performed 15 days after infarction to test the hypothesis that deletion of MMP-9 changed deposition of collagen of the infarcted area (Figure 2). As expected, quantitative analysis for picrosirius red polarization showed that collagen fractional area was significantly greater in the infarcted region than in the noninfarcted region, irrespective of genotype $(64.4 \pm 9.7 \%$ vs. $13.0 \pm 5.5 \% ; P<0.0001 ; n=15)$. Collagen fractional area in the noninfarcted region of the heart was not different between the two groups $(14.7 \pm 5.5 \%$ vs. 11.5 $\pm 5.4 \% ; P=0.28)$. However, MMP-9 KO mice had significantly less collagen accumulation in the infarcted region than did WT mice (collagen fractional area $58.6 \pm 7.8 \%$ vs. $69.6 \pm 8.4 \%$, respectively; $n=15$; $P=0.02)$. In addition to an increase in the total content, the collagen appeared more organized, with thicker fibers in the WT mice than in the MMP-9 KO mice. Moreover, quantitation of collagen showed significantly less collagen accumulation in the MMP-9 KO mice than in WT controls matched by infarct size (WT, $1.09 \pm 0.06$ vs. KO, $0.73 \pm 0.08 \mu \mathrm{g} / \mathrm{ml} \mathrm{KO}$; $P=0.01)$. Collagen type I $\alpha$ gene expression was not different between the groups (collagen type I $\alpha$ GAPDH: WT, $1.0 \pm 0.1$ vs. KO, $0.9 \pm 0.2 ; P=0.82$, arbitrary units; data not shown). Thus, the reduced collagen level in MMP-9 KO animals is consistent with increased collagen degradation.

Immunobistochemistry. The infarcted region contained numerous macrophages identified by immunoreactive Mac3 throughout the infarcted segments (Figure 3). The number of macrophages increased steadily from day 1 to day 15 . At day 15 there was a reduction in macrophage number in MMP-9 KO mice compared with WT mice, as determined by quantitative, computer-assisted image analysis in the infarcted region (positive fractional area of $22.0 \pm 5.3 \%$ in MMP-9 KO mice vs. $34.3 \pm 8.9 \%$ in WT mice; $P=0.013 ; n=11$ ).

Neutrophils increased from day 1 to day 7 , with a greater increase in the WT mice $(31.1 \pm 15.1 \%$ in WT vs. $12.6 \pm 11.1 \%$ in MMP-9 KO; $P=0.16 ; n=6)$. There were no differences in densities of CD4- or CD8-positive cells between MMP-9 KO and WT mice, suggesting that $\mathrm{T}$-cell infiltration was not affected by the absence of MMP-9. Smooth muscle cells immunoreactive for $\alpha$-actin were decreased in microvessels in MMP-9 KO mice (79.8 \pm 28.3 per section) compared with WT mice $(104.1 \pm 23.8$ per section; $P=0.07$; $n=11$ ). A similar trend was found in the number of microvessels detected by CD31 antibody (77.8 \pm 94.7 per section in MMP-9 KO mice vs. $151.2 \pm 44.2$ per section in WT mice; $P=0.10 ; n=11$ ).

Western analysis. MMPs have overlapping substrate specificities, so the effect of loss of specific activities of one enzyme can possibly be reduced by compensation by other enzymes. To test the hypothesis that MMP-9 deletion may affect expression of other MMPs, Western analyses of tissue extracts were performed. Animals

Table 2

Sequential changes in echocardiographic measurements of infarcted mice from day 1 to day 15

\begin{tabular}{|c|c|c|c|c|c|c|}
\hline \multirow[t]{2}{*}{ Measurement } & \multicolumn{2}{|c|}{ Day 4} & \multicolumn{2}{|c|}{ Day 8} & \multicolumn{2}{|c|}{ Day 15} \\
\hline & $\begin{array}{c}\text { MMP-9 KO } \\
n=25\end{array}$ & $\begin{array}{c}\text { WT } \\
n=25\end{array}$ & $\begin{array}{c}\text { MMP-9 KO } \\
n=20\end{array}$ & $\begin{array}{c}\text { WT } \\
n=24\end{array}$ & $\begin{array}{c}\text { MMP-9 KO } \\
n=20\end{array}$ & $\begin{array}{c}\text { WT } \\
n=20\end{array}$ \\
\hline \multicolumn{7}{|l|}{ Midpapillary } \\
\hline$\Delta$ ED diameter $(\mathrm{mm})$ & $0.0 \pm 0.4$ & $0.1 \pm 0.4$ & $-0.1 \pm 0.4^{\mathrm{A}}$ & $0.4 \pm 0.2$ & $0.07 \pm 0.4^{\mathrm{A}}$ & $0.5 \pm 0.4$ \\
\hline$\Delta \mathrm{ES}$ diameter $(\mathrm{mm})$ & $0.05 \pm 0.5$ & $0.0 \pm 0.7$ & $0.0 \pm 0.6^{B}$ & $0.3 \pm 0.5$ & $0.04 \pm 0.5^{C}$ & $0.3 \pm 0.5$ \\
\hline$\Delta \% \mathrm{M}$-mode FS & $-2.0 \pm 14.8$ & $2.5 \pm 19.1$ & $-1.0 \pm 14.7$ & $-2.0 \pm 15.4$ & $0.1 \pm 14.3$ & $0.4 \pm 14.9$ \\
\hline \multicolumn{7}{|l|}{ Apical } \\
\hline$\Delta$ ED diameter $(\mathrm{mm})$ & $0.2 \pm 0.3$ & $0.3 \pm 0.5$ & $0.2 \pm 0.4^{C}$ & $0.5 \pm 0.3$ & $0.3 \pm 0.5^{\mathrm{B}}$ & $0.7 \pm 0.6$ \\
\hline$\Delta \mathrm{ES}$ diameter $(\mathrm{mm})$ & $0.1 \pm 0.5$ & $0.3 \pm 0.6$ & $-0.1 \pm 0.6^{C}$ & $0.5 \pm 0.4$ & $-0.1 \pm 0.7^{D}$ & $0.6 \pm 0.7$ \\
\hline$\Delta \% \mathrm{M}$-mode FS & $1.7 \pm 13.7$ & $-2.0 \pm 14.5$ & $7.0 \pm 15.7^{\mathrm{E}}$ & $-4.0 \pm 11.0$ & $11.2 \pm 18.3^{\mathrm{D}}$ & $-6.0 \pm 17.2$ \\
\hline
\end{tabular}

All measurements are changes compared with baseline measurements. Data are expressed as mean \pm SD. Statistical analyses are MMP-9 KO vs. WT. ${ }^{A} P<0.0005 ;{ }^{B} P \leq 0.05 ;{ }^{C} P \leq 0.01 ;{ }^{D} P<0.005 ;{ }^{E} P=0.06$. 


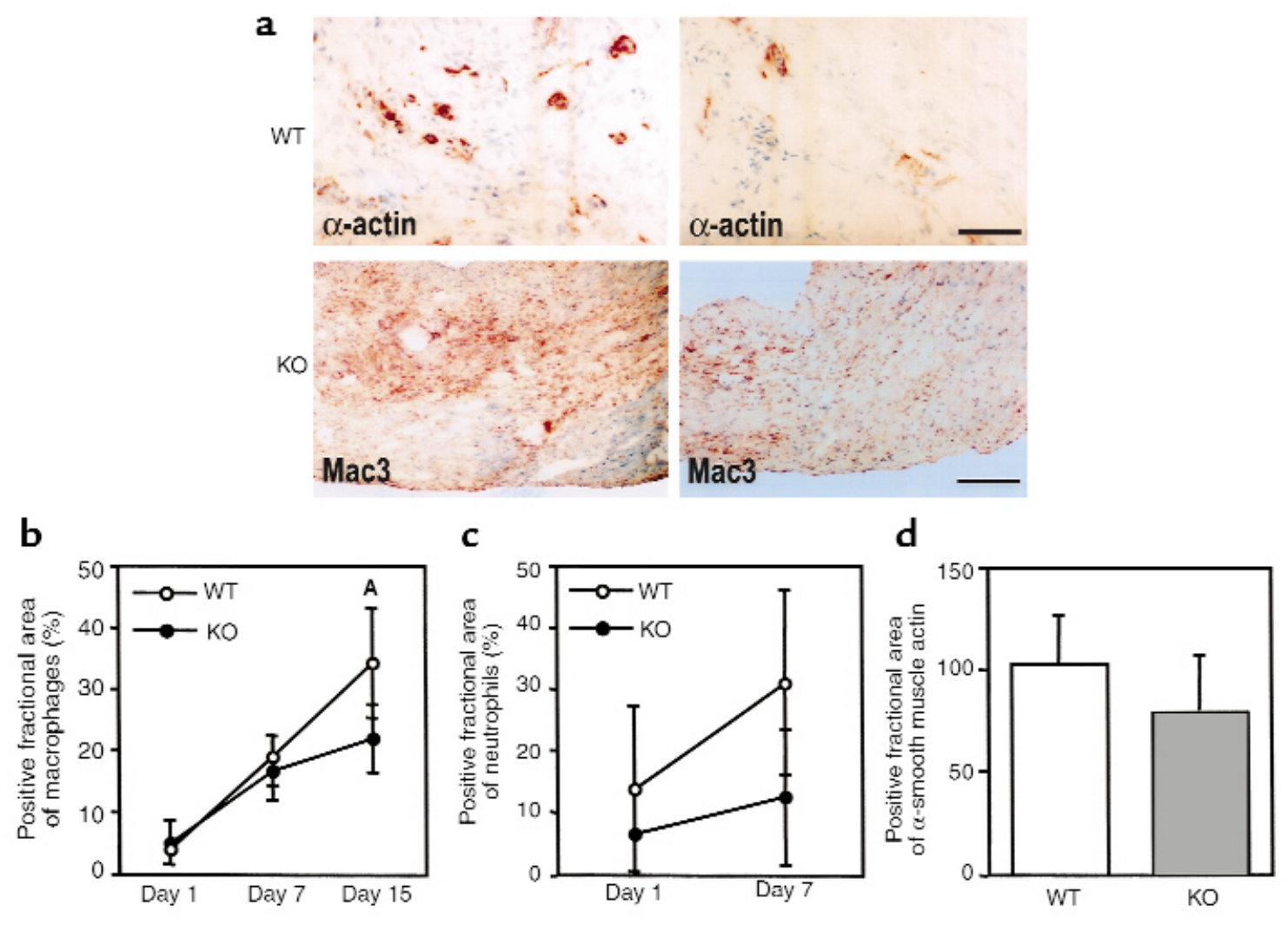

Figure 3

(a) Representative examples of staining of $\alpha$-actin $(\times 400)$ and macrophages (Mac3, $\times 100$; scale bar: $200 \mu \mathrm{m})$ of the infarcted region 15 days after $\mathrm{MI}$, and quantitative fractional areas of $(\mathbf{b})$ macrophages and (c) neutrophils from day 1 to day 7 after $\mathrm{MI}$. ${ }^{\mathrm{A}} P<0.05$. (d) $\alpha$-actin fractional area at day 15 .

that were not operated on showed no differences in the expression of MMP-2 and TIMP-1; however, expression of MMP-3 and MMP-13 were elevated in KO mice (fold increases respectively $1.7 \pm 0.1[P=0.02]$ and $2.1 \pm 0.1$ $[P<0.01])$ (Figure 4). After MI there was increased expression of MMP-2 (fold increase, $2.1 \pm 0.3 ; P<0.05$ ), MMP-3 (fold increase, $1.7 \pm 0.3 ; P=\mathrm{NS}$ ), MMP-13 (fold increase, $3.3 \pm 0.9 ; P<0.05$ ), and TIMP-1 (fold increase, $2.1 \pm 0.3 ; P<0.05)$ in the extracts of MMP-9 KO mice compared with WT mice (Figure 4). These data suggest that MMP-9 may play a role in regulating the expression of other MMPs.

\section{Discussion}

LV enlargement after MI is an important clinical determinant of morbidity and mortality (19-21). This study demonstrated that targeted deletion of the MMP-9 gene attenuates LV enlargement after experimental MI in mice. Differences in collagen accumulation within the infarcted heart, associated with enhanced expression of some MMPs, may contribute to this effect. These data suggest that individual MMPs may be therapeutic targets for LV dilation after MI.

The wound repair process involves temporally overlapping phases, including inflammation, new tissue formation, and tissue remodeling (22). During the inflammatory phase, collagen and other extracellularmatrix components may be degraded. At that time, MMP-9 is expressed primarily in myeloperoxidase-positive or CD45-positive leukocytes and in Mac-3-positive macrophages (10). We did not find any change in echocardiographically detectable LV remodeling in this earliest wound healing phase (at day 4). This observation suggests that MMP-9 does not play a major role in the earliest inflammatory phase. It should be noted that these animals underwent total coronary occlusion, and the influence of MMP-9 on the inflammatory process of ischemia and reperfusion was not evaluated in this study.

By day 15 , there was a decrease in macrophage accumulation in the infarcted region of left ventricles of MMP-9 KO animals. Macrophages have a pivotal role in the transition between inflammation and repair; they participate in the wound-healing process through matrix degradation, neovascularization, and recruitment and proliferation of fibroblasts (22). Macrophages express many growth factors, including PDGF, basic FGF, TGF- $\alpha$ and $-\beta$, and TNF- $\alpha$ (23-26). This finding raises the hypothesis that MMP-9 affects ventricular remodeling through recruitment of macrophages. In contrast, we and others found that neutrophil migration does not require MMP-9 (27). 
MMPs and TIMPs are overexpressed in ventricles of patients and animals with congestive heart failure (4-7). Immunoblotting showed enhanced expression of MMP-2, MMP-13, and TIMP-1 in the infarcted apical region of the MMP-9-deficient mice. Interestingly, even animals that were not operated on showed elevated protein levels of MMP-3 and MMP-13, but not MMP-2 or TIMP-1. The cause for this "compensatory" regulation of other MMPs is not known. Upregulation of MMPs has been reported in MMP-7 KO mice that were not operated on (28). A likely hypothesis is that MMP-9 has an in vivo substrate that can provide specific induction of at least MMP-3 and MMP-13. Because the dominant MMP in vivo substrates are not known, the specific mechanism remains to be determined. In addition, numerous stimuli can promote MMP expression, including reactive oxygen species, changes in cell shape, cytokines, growth factors, and phorbol esters (29). These data suggest that MMP-9 participates in an interacting network of MMP regulation, and that deletion of MMP-9 may lead to enhanced expression of other MMPs that could be partially compensatory.

During morphogenesis, MMPs and TIMPs are spatially and temporally regulated (30). MMPs are prominently overexpressed in the wound-repair process, and are under intense investigation as therapeutic targets for inflammatory arthritis and cancer $(31,32)$. One theme that is emerging from recent studies is that the apparent redundancy of substrates among MMP family members may allow one member of the family to substitute for another during normal processes such as development. In contrast, during pathologic circumstances, deficiency of a single MMP family member may lead to profound consequences $(30,33)$.

An unanticipated finding of this study was decreased accumulation of interstitial collagen in the infarcted region of the MMP-9 $\mathrm{KO}$ animals compared with that in WT siblings. Scar formation involves the interaction of macrophages, myofibroblasts, extracellular matrix components, and cytokines. Collagen remodeling is a dynamic process that depends on continuous synthesis, organization, and catabolism (34). However, because there were no differences in collagen type $1 \alpha$ mRNA, but increased MMP protein content in MMP-9 KO animals after MI, increased collagen degradation is probably responsible for the decreased collagen accumulation in MMP-9 KO animals. Nevertheless, how this decrease in collagen content might influence wall diameters is not clear. Because the chamber diameters depend on many factors, we cannot directly assume that collagen content alone led to the differences in diameter.

Heymans et al. reported that MMP-9-deficient mice have reduced cardiac rupture compared with WT mice (10). A reduction in leukocyte infiltration was observed after MI in MMP-9-deficient animals compared with WT animals. In our study, a trend toward reduced cardiac rupture was noted, but this trend did not reach sta-

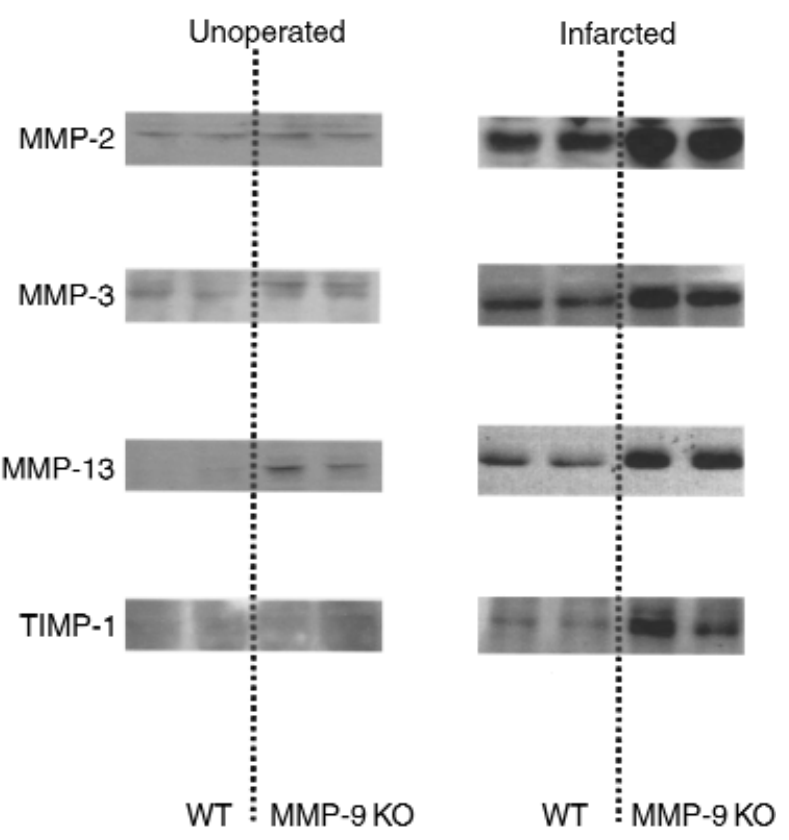

\section{Figure 4}

Western analysis for MMP-2, MMP-3, MMP-13, and TIMP-1 in animals that were not operated on (left panel) or 15 days after MI (right panel). MMP-9 KO mice had increased expression of these MMPs and TIMP-1 compared with WT mice.

tistical significance. One difference between the Heyman study and our study is that our WT rupture rate was much lower, possibly due to surgical technique. Because we used sibling controls of heterozygous mating pairs and attempted to achieve a uniform genetic background by at least six backcrosses, it is unlikely that background effects between the MMP-9 KO and WT groups influenced the results of our study.

The attenuated dilation, decreased collagen accumulation, and reduced early cardiac rupture associated with deletion of MMP-9 are theoretically beneficial events; future studies may determine the potential of MMP-9 as a therapeutic target. The persistence of inflammation at 15 days in our study demonstrates that the inflammatory phase was not yet complete, so it is possible that MMP-9 KO animals may have either accelerated or reduced dilation after this time. Our study was not designed to address the long-term functional or survival implications of MMP-9 or LV functional properties. These data suggest, however, that MMPs actively participate in remodeling ventricular tissues after MI, and that specific MMPs have prominent roles in this process.

\section{Acknowledgments}

This work was supported by the McLaughlin Foundation (Canada) and the Deutsche Forschungsgemeinschaft. The authors thank Christopher C. Hill for expert technical assistance. 
1. Pfeffer, J.M., Pfeffer, M.A., Fletcher, P.J., and Braunwald, E. 1991. Progressive ventricular remodeling in rat with myocardial infarction. Am.J. Physiol. 260:H1406-H1414.

2. Rumberger, J.A. 1994. Ventricular dilatation and remodeling after myocardial infarction. Mayo Clin. Proc. 69:664-674.

3. Tyagi, S.C., Ratajska, A., and Weber, K.T. 1993. Myocardial matrix metalloproteinase(s): localization and activation. Mol. Cell. Biochem. 126:49-59.

4. Cleutjens, J.P., Kandala, J.C., Guarda, E., Guntaka, R.V., and Weber, K.T. 1995. Regulation of collagen degradation in the rat myocardium after infarction. J. Mol. Cell. Cardiol. 27:1281-1292.

5. Cleutjens, J.P., Verluyten, M.J., Smiths, J.F., and Daemen, M.J. 1995. Collagen remodeling after myocardial infarction in the rat heart. Am. J. Pathol. 147:325-338.

6. Thomas, C.V., et al. 1998. Increased matrix metalloproteinase activity and selective upregulation in LV myocardium from patients with endstage dilated cardiomyopathy. Circulation. 97:1708-1715.

7. Coker, M.L., et al. 1998. Myocardial matrix metalloproteinase activity and abundance with congestive heart failure. Am. J. Physiol. 274:H1516-H1523.

8. Spinale, F.G., et al. 1999. Matrix metalloproteinase inhibition during the development of congestive heart failure: effects on left ventricular dimensions and function. Circ. Res. 85:364-376.

9. Rohde, L.E., et al. 1999. Matrix metalloproteinase inhibition attenuates early left ventricular enlargement after experimental myocardial infarction in mice. Circulation. 99:3063-3070.

10. Heymans, S., et al. 1999. Inhibition of plasminogen activators or matrix metalloproteinases prevents cardiac rupture but impairs therapeutic angiogenesis and causes cardiac failure. Nat. Med. 5:1135-1142.

11. Vu, T.H., et al. 1998. MMP-9/gelatinase B is a key regulator of growth plate angiogenesis and apoptosis of hypertrophic chondrocytes. Cell. 93:411-422.

12. Michael, L.H., et al. 1995. Myocardial ischemia and reperfusion: a murine model. Am. J. Physiol. 269:H2147-H2154.

13. Junqueira, L.C., Bignolas, G., and Brentani, R.R. 1979. Picrosirius staining plus polarization microscopy, a specific method for collagen detection in tissue sections. Histochem. J. 11:447-455.

14. Aikawa, M., et al. 1998. Lipid lowering by diet reduces matrix metalloproteinase activity and increases collagen content of rabbit atheroma: a potential mechanism of lesion stabilization. Circulation. 97:2433-2444.

15. Marotta, M., and Martino, G. 1985. Sensitive spectrophotometric method for the quantitative estimation of collagen. Anal. Biochem. 150:86-90.

16. Walsh, B.J., Thornton, S.C., Penny, R., and Breit, N. 1992. Microplate reader-based quantitation of collagens. Anal. Biochem. 203:187-190.

17. Lee, R.T., Schoen, F.J., Loree, H.M., Lark, M.W., and Libby, P. 1996. Circumferential stress and matrix metalloproteinase 1 in human coronary atherosclerosis. Implications for plaque rupture. Arterioscler. Thromb. Vasc. Biol. 16:1070-1073.
18. Brilla, C.G., Pick, R., Tan, L.B., Janicki, J.S., and Weber, K.T. 1990. Remodeling of the rat right and left ventricles in experimental hypertension. Circ. Res. 67:1355-1364.

19. White, H.D., et al. 1987. Left ventricular end-systolic volume as the major determinant of survival after recovery from myocardial infarction. Circulation. 76:44-51.

20. Kostuk, W.J., Kazamias, T.M., Gander, M.P., Simon, A.L., and Ross, J.J. 1973. Left ventricular size after acute myocardial infarction. Serial changes and their prognostic significance. Circulation. 47:1174-1179.

21. Pfeffer, M.A., and Braunwald, E. 1990. Ventricular remodeling after myocardial infarction. Experimental observations and clinical implications. Circulation. 81:1161-1172.

22. Singer, A.J., and Clark, R.A. 1999. Cutaneous wound healing. N. Engl. J. Med. 341:738-746.

23. Rappolee, D.A., Mark, D., Banda, M.J., and Werb, Z. 1988. Wound macrophages express TGF-alpha and other growth factors in vivo: analysis by mRNA phenotyping. Science. 241:708-712.

24. Assoian, R.K., et al. 1987. Expression and secretion of type beta transforming growth factor by activated human macrophages. Proc. Natl. Acad. Sci. USA. 84:6020-6024.

25. Leibovich, S.J., et al. 1987. Macrophage-induced angiogenesis is mediated by tumour necrosis factor-alpha. Nature. 329:630-632.

26. Leibovich, S.J., and Ross, R. 1975. The role of the macrophage in wound repair. A study with hydrocortisone and antimacrophage serum. Am.J. Pathol. 78:71-100.

27. Betsuyaku, T., Shipley, J.M., Liu, Z., and Senior, R.M. 1999. Neutrophil emigration in the lungs, peritoneum, and skin does not require gelatinase B. Am. J. Respir. Cell Mol. Biol. 20:1303-1309.

28. Rudolph-Owen, L.A., Hulboy, D.L., Wilson, C.L., Mudgett, J., and Matrisian, L.M. 1997. Coordinate expression of matrix metalloproteinase family members in the uterus in normal, matrilysin-deficient, and stromelysin-1-deficient mice. Endocrinology. 138:4902-4911.

29. Nagase, H., and Woessner, J.F. 1999. Matrix metalloproteinases. J. Biol. Chem. 138:4902-4911.

30. Werb, Z., and Chin, J.R. 1998. Extracellular matrix remodeling during morphogenesis. Ann. NY Acad. Sci. 857:110-118.

31. Edwards, D.R., and Murphy, G. 1998. Cancer. Proteases: invasion and more. Nature. 394:527-528.

32. Liu, Z., et al. 1998. Gelatinase-B-deficient mice are resistant to experimental bullous pemphigoid. J. Exp. Med. 188:475-482.

33. Shapiro, S.D. 1998. Matrix metalloproteinase degradation of extracellular matrix: biological consequences. Curr. Opin. Cell Biol. 5:602-608.

34. Madlener, M., Parks, W.C., and Werner, S. 1998. Matrix metalloproteinases (MMPs) and their physiological inhibitors (TIMPs) are differentially expressed during excisional skin wound repair. Exp. Cell Res. 242:201-210. 